\title{
De la «sujétion», ou Crise de la sémiotique ${ }^{1}$
}

\author{
Per Aage Brandt' \\ https://orcid.org/0000-0001-7774-5033 \\ I - Case Western Reserve University \\ Cleveland, EUA
}

Résumé: Est-ce que la sémiotique peut théoriser la subjectivité? Etant donné que tout signe communiqué s'inscrit dans un cadre énonciatif, l'inter-subjectivité est présupposée dans la sémiosis, dont elle structure la relation déictique; mais la subjectivité telle quelle, qui relève de la psychologie cognitive et de la phénoménologie, tant existentielle que formelle, est-elle toujours à considérer comme une entité appartenant au domaine du sens et de la sémiosis? Dans cette note, nous avançons l'hypothèse que les sémioses gravitent autour d'une colonne allant du sens symbolico-performatif au sens iconico-libidinal, en passant par le sens cogito-épistémique lié au diagrammatisme. Ce système psycho-sémiotique organise la subjectivité en l'orientant vers la stratification écologique de la socio-sphère, qui passe par les mêmes instances sémiotiques, et vers l'inter-subjectivité communicante. L'occasion d'esquisser cette architecture psychosémiotique nous a été offerte par un article, maintenant dé-publié, qui dérivait la subjectivité d'un parcours génératif sémiotique, et qui se trouvait, à notre humble avis, en difficulté par rapport à la cognition et la corporéité qui doivent, ontologiquement, fonder le sujet.

Mots clefs: cogito; critique; libido; subjectivité; sujet.

Abstract: Of "subjection", or Crisis of semiotics - Can semiotics theorise subjectivity? Since all communicated signs are framed by enunciation, inter-subjectivity is presupposed in semiosis, because it structures its deictic relations; but can subjectivity as such - which is a matter of cognitive psychology and phenomenology, both existential and formal - still be considered as an entity pertaining to the domain of meaning and semiosis? In this note, I present the hypothesis that semioses of different kinds gravitate around a constitutive column of meaning-making spanning from perception and symbolic-performative meaning through cogito-epistemic and diagrammatic meaning to memory and iconic-libidinal meaning. This psycho-semiotic system organizes subjectivity and orients it towards the ecological

1 Une première version du présent article a été publiée le 28 février 2020 dans la revue Actes Sémiotiques, 123, 2020, mais en a été éliminée par la nouvelle direction mise en place le 4 mars, en même temps qu'était censuré I'ensemble de la rubrique où elle figurait (LANDOWSKI, 2020). Ce même numéro 123 présente également un article, quant à lui non censuré, de l'auteur sur la subjectivité vue sous l'angle de l'énonciation. 
stratification of the socio-sphere, which offers the same semiotic instances, and towards communicative intersubjectivity. The opportunity to outline this psycho-semiotic architecture was given by a later de-published article in which subjectivity was derived from the semiotic generative process, and which according to my view was in trouble, as a constitution of the subject, due to its lack of ontological connections to studies of cognition and the embodied mind.

Keywords: cogito; critique; libido; subjectivity; subject.

Resumo: Da "sujeição", ou Crise da semiótica - Pode a semiótica teorizar a subjetividade? Considerando que todo signo comunicado se inscreve em um quadro enunciativo, a intersubjetividade é pressuposta na semiose, daí ela estrutura a relação dêitica; mas será que a subjetividade tal qual, que depende da psicologia cognitiva e da fenomenologia, tanto existencial quanto formal, pode ainda ser considerada como pertencente ao domínio do sentido e da semiosis? Nesta nota, avançamos a hipótese que as semioses gravitam em torno de uma coluna indo do sentido simbólico-performativo até o sentido icônico-libidinal, passando pelo sentido cogito-epistêmico ligado ao diagramatismo. A oportunidade de esboçar esta arquitetura psico-semiótica foi-nos ofertada por um artigo, agora des-publicado, que derivava a subjetividade de um percurso gerativo semiótico, e que se encontrava, em nosso humilde ponto de vista, com problemas em relação à cognição e a corporeidade que devem ontologicamente fundar o sujeito.

Palavras-chave: cogito; crítica; libido; subjetividade; sujeito.

\section{Avant-propos}

A quelles conditions la sémiotique de tradition française fondée sur le travail théorique d'Algirdas J. Greimas peut-elle prendre en charge la problématique complexe, phénoménologique, sociale et psychologique de la subjectivité? Le texte qui suit a eu pour point de départ la lecture d'un article inédit de Pierluigi Basso Fossali portant précisément sur la constitution sémiotique de la subjectivité. L'ambitieux projet de cet auteur, reposant sur une réinterprétation du parcours génératif de Greimas, vise à construire un parcours constitutif du sujet, une «subjectiv-isation», pourrait-on dire, qu'il appelle (néologiquement) sujétion. Malgré une rédaction parfois difficile à déchiffrer, ce texte à paraître a appelé de ma part une considération attentive. D'où la critique à la fois méthodologique et ontologique qu'on va lire.

\section{Quatre points critiques}

Premier point. Les tenants de la sémiotique structurale cultivent l'immanentisme venu de Louis Hjelmslev, qui pensait que la linguistique, comprise comme méthode glossématique, était le métalangage de toute science et de toute philosophie, parce que pour lui, le langage et la pensée coïncident. La linguistique ne devait donc dépendre d'aucune discipline existant en dehors d'elle. Et puisque la linguistique ainsi comprise 
constituait le métalangage de tous les langages (ou sémiotiques) objets, on pouvait croire que la connaissance du langage donnait un accès privilégié à tout problème scientifique, philosophique ou autre. L'immanentisme proclamé par les sémioticiens opérant dans cette ligne fait que l'on essaie de se passer de toute autre source de savoir que celle qui découle de l'analyse du langage et des concepts qui s'y sont développés. Or le langage n'est pas la pensée, et par suite la linguistique n'est qu'une discipline parmi d'autres, et aucunement un métalangage - concept analytique qui n'a en l'occurrence aucune justification ${ }^{2}$.

Deuxième point. Un autre problème auquel se heurte la sémio-linguistique est celui qu'entraîne l'habitude de croire que la sémantique textuelle et structurale de Greimas et de ses collaborateurs constitue une véritable linguistique, au sens de Saussure ou de Hjelmslev, ou de quiconque, et en même temps une sémiotique, au sens de Peirce, alors qu'il s'agit en réalité d'une analyse littéraire généralisée, ce qui est déjà beaucoup, mais tout à fait autre chose. Cette «sémiotique» qui est une sémantique textuelle contient notamment des ébauches de narratologie et de rhétorique, mais elle ne s'occupe nullement de ce qui intéresse les linguistes, à savoir le fonctionnement des langues, de leurs syntaxes, de leurs morphologies, des principes de leurs grammaires, de leurs typologies, de leurs généalogies, de leurs phonétiques, de leur fondement biologique et de leur évolution, de leurs rôles dans la cognition, etc. Elle ne participe pas au débat concernant les multiples théories du langage existantes. Par ailleurs, elle commente souvent les arts, la poésie, mais elle n'a jamais repris, après les ébauches de Lotman et de Jakobson, les tentatives de classification et de théorisation des systèmes de signes, des gestes, des médias - et, d'une manière générale, des fonctions sémiotiques repérables dans le monde culturel et social. Elle continue à faire de la sémantique textuelle, "discursive», en la généralisant, comme si le monde était un grand texte. Mais le monde n'est pas un grand texte. En revanche, il serait intéressant de comprendre sur quelle sphère repose la sémio-sphère. Je dirais: la sphère de la planète sur laquelle nous vivons, le monde de I'humanité que constituent à la fois I'ensemble des sociétés et I'ensemble des conditions naturelles, matérielles, écologiques, de notre existence planétaire (ELLIS, 2019).

Troisième point: Le statut épistémique de cette sémiotique et de son «discours». Elle n’a jamais ni cherché, ni par suite réussi, à clarifier son rapport à la philosophie. Est-ce qu'une sémiotique générale selon ces lignes serait une philosophie - par exemple une phénoménologie? ${ }^{3}$, une philosophie analytique comme celle de Hjelmslev? Ou serait-elle une sorte de science, ou bien autre chose encore, par exemple une agglomération de disciplines? Ou simplement un ensemble de modèles, de méthodes et de diagrammes heuristiques? La philosophie sémio-physique de RenéThom pourrait être à cet égard une perspective à explorer

2 Sur ce point, je recommande l'ouvrage de Sémir Badir (2014). Il conteste vivement la validité de la position que je défends ici.

3 Le volume monumental rédigé par Dan Zahavi (2012), ne contient aucune référence à la sémiotique. Sur l'intentionnalité, en revanche, il est évidemment fort généreux.

4 Une sémiotique dynamique, voire morpho-dynamique, a ainsi été développée par Jean Petitot, Wolfgang Wildgen (2010) et l'auteur, entre autres, dans la ligne de la pensée du mathématicien-philosophe français. 
Quatrième point: La question de l'ontologie. Le sens, s'il existe, doit se situer à la base du social, du communicationnel et du psychique - du subjectif, si on veut. Or ces trois blocs ontologiques (qu'est-ce qu'une société, qu'est-ce qu'une communication, qu'estce qu'un psychisme?) ont fait l'objet de recherches, de débats et de théorisations plus ou moins scientifiques depuis des siècles, sans que la sémiotique apparemment se sente concernée par les questions brûlantes émergeant de la masse de travaux qui en témoigne. Exemples, questions pour une sémiotique: Si le modèle de Marx reste à considérer, où est alors l'écologie terrestre dans cette perspective, et où en est la sémiotique de l'argent? Si le modèle linguistique de Noam Chomsky ou celui de Ronald Langacker restent plausibles (pour ne pas parler des grammaires constructionnelles), où se situe alors le «parcours génératif» sémio-linguistique? Si les modèles psychologiques de Freud et de ses écoles, par exemple la lacanienne, sont toujours au programme, où se trouve alors l'énonciation?

Tout cela reste à élaborer et surtout à resituer dans le paysage des recherches contemporaines en dehors de la sémiotique, en anthropologie, en sciences cognitives, en psychologie ou en psychiatrie, et même en économie, en médecine, en sciences politiques. Vaste programme, vu le retard. Ensuite, on pourrait mieux discuter le concept de sujet.

\section{Le «sujet», être de langage ou être de chair? ${ }^{5}$}

Est-ce que nous pouvons oser, avec nos moyens limités, esquisser malgré tout une architecture du sujet compatible avec nos notions de sémiotique?

Il faut commencer par Descartes: le cogito, une pensée à la première personne. Le sujet y est - opposé à l'objet, comme il se doit. Il sera bientôt corrigé par Spinoza, dont le /cogitat/ à la troisième personne a pour sujet le deus sive natura. Exit le sujet: il n'est rien, car il est tout. Dans le monisme de Spinoza, tout pense, tout est comportement qui pense. Le thème (celui de Basso Fossali, après Fontanille) de la cavité corporelle comme subjectivité pourrait bien être spinoziste, en ce sens (car il y a des cavités partout), alors que la problématique de l'énonciation pourrait plaire à l'auteur du Discours de la méthode, dans la mesure où son je doute exprime une réflexivité épistémique qui a effectivement besoin de la première personne. C'est bien Descartes qui fonde la phénoménologie. On aurait ainsi deux histoires complémentaires pour commencer. Le sujet a un corps; mais il n'est pas un corps. C'est une chair qui doute.

Le cogito aurait pourtant besoin d'une libido augustinienne pour ressembler à un sujet ${ }^{6}$. Le cogito est géomètre, alors que la libido est plutôt peintre, souvent surréaliste. Ce sont là deux sémiotiques différentes, et deux régimes du sens, I'un épistémique, I'autre affectif.

5 «Greimas (FIORIN, 2017) conceives of enunciation in a radical, anti-metaphysical manner. This does not mean that, for him, there is no real enunciation. For him, the issue is that one can talk in terms of real enunciation only because it is projected in the text, creating a methodological simulacrum that allows talking about enunciative processes and operations».

6 Pour s'en rassurer, consulter le dernier livre de Michel Foucault (2018), et surtout ses interprétations de Saint Augustin, qui expliquent comment devenir un sujet, via la libido. 
Une sémiotique mathématico-diagrammatique, dirais-je, et une sémiotique iconique. On voit bien ce qui manque alors: le rapport au pouvoir, un agito actantiel et une sémiotique symbolique correspondante. Les expressions symboliques en effet déclarent, promettent, menacent, instruisent, ordonnent, agissent en créant des états sociaux. Cogito, libido, agito: on a ainsi au moins trois étages de la subjectivité (BRANDT, 2020). Du même coup, on a aussi trois histoires, et corrélativement, trois types de sens: I'un épistémique ou cogitatif (logique), l'autre libidinal (affectif), le troisième agitatif (performatif). Il est clair, pourtant, que si ces composantes sont à insérer entre la perception et la mémoire à long terme, comme il se doit dans une théorie réaliste, l'ordre n'est pas aléatoire. La perception est au plus près de l'agitatif, parce que notre attention se porte primairement sur la situation en termes de possibilités d'agir. D'autre part, comme la mémoire se sert de marques affectives pour permettre à la conscience de rappeler les contenus mémorisés, il faut que le cogitatif s'insère entre l'agitatif et le libidinal (au sens large) ${ }^{8}$. On obtient ainsi une petite topologie du sujet:

Le sujet entouré de ses sémiotiques -

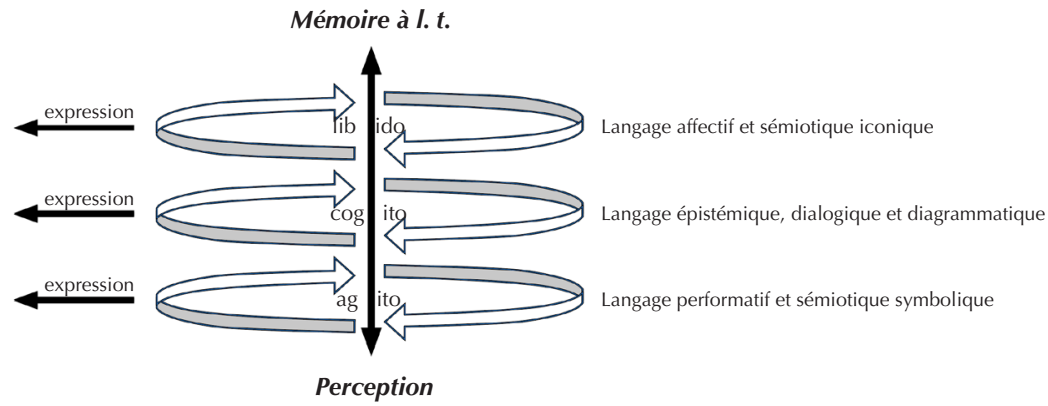

Fig. 1. Topologie du sujet.

L'affectif et le performatif risquent constamment d'éclipser l'épistémique, ce qui engendre un comportement impulsif «irréfléchi», «étourdi».

La problématique du pouvoir, qui concerne les multiples références à la «résistance» dans I'article que je commentais, peut être abordée par celle de l'énonciation. Quand Emile Benveniste déclare que c'est dans et par le langage que I'homme se constitue comme sujet, il pense d'abord à l'énonciation telle que manifestée par le jeu des pronoms personnels. Or le jeu des «personnes», le je, le tu et la tierce instance, s'il est bien présent dans toutes les langues connues, relève probablement d'un fonctionnement encore plus profond que le langage où il se manifeste. Il existe chez beaucoup d'animaux (oiseaux, primates). C'est dans l'échange intersubjectif même, dans notre capacité à être

7 Ou si on préfère (plus au goût du jour peut-être, mais moins précis), «agentif».

8 Le rôle (JACOB, 1999) des marqueurs somatiques émotionnels a été remarqué par le psychologue cognitif Antonio Damasio, dont on doit connaître surtout. 
attentifs à l'attention de l'autre et non seulement aux choses, qu'on peut voir l'origine du dialogue, où le je-tu réversible se déploiera.

Dans l'agito, comme dans la libido, ce jeu réversible cède la place à d'autres réciprocités. Dans l'action et l'usage performatif du langage, il s'agit plutôt de quelque chose comme le jeu (game, Spiel) au sens de Wittgenstein. Et dans l'échange affectif, c'est le mimétisme qui domine. L'énonciation est donc le plus déployée, et elle est peut-être née, au niveau du cogito, alors qu'elle s'affaiblit en même temps que lui, par exemple dans des états d'amour, de fureur ou d'intoxication. La structure fondamentale de l'énonciation comme dispositif est ainsi un schéma ternaire par lequel s'établit le lien entre deux sujets et un objet d'attention que l'un montre à l'autre: je te le donne à voir. $C^{\prime}$ est un rapport déictique. II s'agit d'attention, de deixis et de don. Le don de parole se fonde sur le principe d'un dire-vrai: on s'adresse en principe à l'autre au nom de la vérité, car le cogito présuppose que penser veut dire penser aussi vrai que possible.

En revanche, dans le registre agitatif, on s'adresse à l'autre au nom d'une puissance. La puissance demande l'obéissance, et elle menace ou instruit ; elle dit: si tu n'obéis pas, on punit ton corps ou ton nom, ta dignité (on affaiblit ton identité). Pourquoi? Parce que c'est comme ça, dit-elle. Pourquoi encore? Parce qu'on a la force physique qui légifère, ou qu'on possède la sainteté qui doit te reconnaître pour que tu existes et aies un nom parmi les tiens. La loi et le nom, on peut résumer ainsi les principes profonds derrière la puissance, ceux qui la rendent possible. Parler de la puissance en de tels termes, c'est aussi parler de la légalité qui est à la base de toute égalité, et qui situe le citoyen lambda comme... lambda, sans plus, sans différence. Mais c'est d'autre part parler de l'identité qui fait la différence, et de la sainteté de l'identitaire communautaire qui lutte pour sa distinction aux yeux de tous. La singularité du sujet s'interprète dans le sens de l'identité quantitative (on n'est qu'un 1, lambda), et dans celui de l'identité qualitative (on est quelque chose, et on en est surtout très fier).

\section{Une subjectivité critique}

Je me demande régulièrement comment comprendre la possibilité d'une subjectivité critique, capable de résister à la séduction libidinale comme à la violence agitative, de rester à l'écart et maintenir le doute en face des certitudes. D'où vient, donc, la possibilité de la critique?

Pour tenter d'y répondre, je devrais sans doute supposer qu'à côté des modes constitutifs du subjectif - cogito, libido, agito - il en existe un quatrième qui les renforcerait tous. C'est en effet possible. Regardons le cogito dialogique, à trois positions énonciatives bien déployées: je te parle / tu me parles / de ce qui nous concerne tous. Dans le mimétisme libidinal du rapport de séduction, cela se réduit à deux positions déployées, je et $t u$ : je t'aime / tu m'aimes / et rien d'autre ne nous importe vraiment. 
La troisième personne, le monde et les autres, s'éclipsent. Finalement, dans un rapport de pouvoir, agitatif, c'est la première personne qui est paralysée: tu dois $x$ (selon la loi), tu es $y$ (selon la religion), tu dois $x$ parce que tu es $y$, et tu es $x$ parce que tu dois $y$, et moi je n'y peux rien (combinaison des deux autorités, et moi je m'excuse). L'énonciateur s'est pétrifié comme un livre, un dieu, ou un officier. On ne peut plus lui parler; la relation est devenue binaire; ainsi dans l'impératif: fais ceci! (il y a toi et ceci, rien d'autre; I'énonciataire est supposé faire et se taire $)^{9}$.

Or il existe un quatrième mode d'énonciation où c'est la deuxième personne qui s'éclipse, ou qui disparaît momentanément. Il y a tout le monde et moi: c'est le cas dans la performance artistique, dans le discours philosophique, comme dans la musique et comme pour le narrateur solitaire dans la fiction. Ce sont des situations énonciatives difficiles à vivre pour la plupart d'entre nous, car on «s'expose», et on est «seul». Mais cette conjoncture énonciative, qui est aussi celle de l'écriture au sens fort, est celle qui est assumée par le sujet critique $^{10}$. La singularité souvent créatrice de la voix qui en résulte semble due à l'absence, ou à la mise en sourdine, de la deuxième personne. Je suis libre parce que tu ne m'entends pas, tu ne filtres plus mon propos! Sinon, tu m'arrêterais bientôt ou immédiatement ${ }^{11}$. C'est une sorte de cogito radical, peut-on dire ${ }^{12}$. La subjectivité critique et résistante, celle du je dans le doute radical, est celle de l'art, de l'écriture, de la pensée radicale, et elle est, à la limite, voisine de la psychose naissante ${ }^{13}$. Le sujet critique est toujours un sujet en crise, dans un certain sens. Ce qui me semble une bonne description.

La sémiotique, elle aussi, est en crise, si elle se croit seule devant le monde du sens, ayant perdu sa capacité de dialogue avec les disciplines du savoir qui l'entourent. Si elle croit que la subjectivité, la notion sans doute la plus importante de toutes celles qui fondent la possibilité du sens, ne concerne qu'elle. Espérons ne pas avoir à y voir le début d'une psychose. Si l'état devient chronique, il n'est pas recommandable.

9 En termes landowskiens (2012), c'est tout à fait le régime de l' «assentiment» à l'accidentel ou à l'arbitraire, à l'absolu du pouvoir (mondain ou «de droit divin»), au fatal.

10 Car le lecteur n'est pas là, comme un interlocuteur, il a été remplacé par un «Lector in Fabula», comme le dit Eco (1985). Cela est différent pour chaque lecteur réel, chacun étant susceptible de se réinstaller comme deuxième personne en se demandant ce que le texte «veut dire», et de s'exprimer graphiquement dans les marges du livre.

11 Je pense ici à Sganarelle dans le Dom Juan de Molière (1665). Il essaie d'argumenter par syllogismes, et quand il sent qu'il s'égare, il demande à son maître de l'arrêter, de l'interrompre avant la catastrophe.

12 Cette idée me vient d'un psychiatre français, Henri Grivois, qui décrit avec beaucoup de verve et d'intuition les grandes composantes — «concernement» et «centralité» — de cette condition dramatique dans laquelle le sujet fait l'expérience de se trouver brusquement au centre de l'attention de tout le monde, ou inversement d'être intensément concerné par toute l'humanité au point de presque disparaître lui-même (GRIVOIS, 1995; BRANDT, 2020).

13 La crise psychotique arrive bien avant les délires thématiques, qui fixeront et bloqueront l'expérience mais resteront à vrai dire incompréhensibles sans la structure initiale. La formation initiale est purement intentionnelle et non thématique; elle est une dynamique d'attentions intersubjectives, raison pour laquelle elle reste très difficile à décrire. Or, dans notre conception de l'énonciation, il s'agit précisément d'une telle formation intentionnelle entre les instances, les «personnes» mentales (en anglais de linguiste, personhood) qui déterminent le sujet. 
Per Aage Brandt é professor e pesquisador da Case Western University.

pabrandt18@gmail.com

\section{Références}

BADIR, S. Epistémologie sémiotique. La théorie du langage de Louis Hjelmslev. Paris: Honoré Champion, 2014.

BRANDT, P. A. What is a global citizen?. In: ELLIS, M. (éd.). Critical Global Semiotics: Understanding Sustainable Transformational Citizenship. Londres: Routledge, 2019.

«La dynamique énonciative de la subjectivité». Actes Sémiotiques, 123, 2020.

DAMASIO, A. Le sentiment même de soi. Corps, émotions, conscience. Paris: Odile Jacob, 1999. (The Feeling of What Happens. Body and Emotion in the Making of Consciousness, Londres, Heinemann, 1999).

ECO, U. Lector in Fabula. Paris: Grasset, 1985.

FIORIN, J. L. Two concepts of enunciation. Semiotica, 2017.

FOUCAULT, M. Les aveux de la chair. Paris: Gallimard, 2018.

LANDOWSKI, E. Shikata ga nai ou Encore un pas pour devenir sémioticien ! Lexia, 11-13, 2012.

éd., Dialogue: Comment penser, comment écrire la sémiotique ? Actes Sémiotiques, 123, 2020 - dossier censuré.

MOLIÈRE. Dom Juan, 1665.

WILDGEN, W. et BRANDT, P. A. (eds.). Semiosis and Catastrophes. René Thom's Semiotic Heritage. Berne, Peter Lang (coll. Sémiotiques Européennes, 10), 2010.

ZAHAVI, D. The Oxford Handbook of Contemporary Phenomenology. Oxford: Oxford University Press, 2012.

Artigo recebido em 05/03/2020

e aprovado em 20/04/2020. 